International Journal of Pure and Applied Mathematics

Volume 95 No. 1 2014, 57-67

ISSN: 1311-8080 (printed version); ISSN: 1314-3395 (on-line version)

url: http://www.ijpam.eu

doi: http://dx.doi.org/10.12732/ijpam.v95i1.7

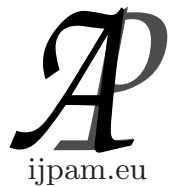

\title{
AN IMPROVED EXPONENTIAL STABILITY OF SWITCHED NEURAL NETWORKS WITH INTERVAL TIME-VARYING DELAY
}

\author{
Manlika Rajchakit ${ }^{1}$, Grienggrai Rajchakit ${ }^{2} \S$ \\ ${ }^{1,2}$ Department of Mathematics and Statistics \\ Maejo University \\ Chiangmai, 50290, THAILAND
}

\begin{abstract}
This paper studies the problem for exponential stability of switched neural networks with interval time-varying delay. The time delay is a continuous function belonging to a given interval, but not necessary to be differentiable. By constructing a set of augmented Lyapunov-Krasovskii functional combined with Newton-Leibniz formula, a switching rule and switching design for exponential stability for of switched recurrent neural networks with interval time-varying delay is designed via linear matrix inequalities, and new sufficient conditions for the exponential stability of switched recurrent neural networks with interval time-varying delay via linear matrix inequalities(LMIs).
\end{abstract}

AMS Subject Classification: 92B05, 93D20

Key Words: neural networks, switching design, exponential stability, interval time-varying delays, Lyapunov function, linear matrix inequalities

\section{Introduction}

Switched systems, typically, composed of a finite number of subsystems and a corresponding switching signal governing the switching law between the subsystems, are a important class of hybrid systems. The motivation for studying

Received: January 20, 2014

(C) 2014 Academic Publications, Ltd.

$\S$ Correspondence author url: www.acadpubl.eu 
switched systems comes from the fact that many practical plants are inherently multi-model in the sense that several dynamic subsystems are needed to describe their behaviour when the system undergoes internal or external abrupt changing of environmental factors . Due to aforementioned characteristics, switched systems have attracted increasing interest in recent years from various fields, such as power electronics and systems, flight control systems, network control systems. Switched systems have been extensively investigated and have produced many sound and pioneered results. In particular, stability problem and switching law design are focuses for switched systems.

As is well known, stability, especially exponential stability, is a primary problem in the science and engineering fields. Switched systems, without exception, possess stability analysis problems. In a certain sense, switched systems can be categorized as state-controlled, time-controlled, or a combination of the two. In recent years, much effort has been focused on time-controlled switched systems. Fortunately, there have been many remarkable results in time-controlled switched systems. A typical example is the average dwell time (ADT), which is demonstrated to be a successful and effective technique to analyse switched system stability and design controllers. Recently, some researchers have studied switched systems with time delay or under a networked environment, and have presented sufficient conditions for stability analysis of switched delay systems under ADT switching signals.

Stability and control of recurrent neural networks with time delay has been attracted considerable attention in recent years [1-5]. In many practical systems, it is desirable to design neural networks which are not only asymptotically or exponentially stable but can also guarantee an adequate level of system performance. In the area of control, signal processing, pattern recognition and image processing, delayed neural networks have many useful applications. Some of these applications require that the equilibrium points of the designed network be stable. In both biological and artificial neural systems, time delays due to integration and communication are ubiquitous and often become a source of instability. The time delays in electronic neural networks are usually time-varying, and sometimes vary violently with respect to time due to the finite switching speed of amplifiers and faults in the electrical circuitry. The Lyapunov-Krasovskii functional technique has been among the popular and effective tool in the design of guaranteed cost controls for neural networks with time delay. Nevertheless, despite such diversity of results available, most existing work either assumed that the time delays are constant or differentiable [6-10]. To the best of our knowledge, a switching rule, switching design and exponential stability for switched neural networks with interval time-varying 
delay, non-differentiable time-varying delays have not been fully studied yet (see, e.g., $[2-5,7-15]$ and the references therein), which are important in both theories and applications. This motivates our research.

In this paper, we investigate the exponential stability for switched neural networks problem. The novel features here are that the delayed neural network under consideration is with various globally Lipschitz continuous activation functions, and the time-varying delay function is interval, non-differentiable. Based on constructing a set of augmented Lyapunov-Krasovskii functional combined with Newton-Leibniz formula, new delay-dependent exponential stability criteria for switched neural networks with interval time-varying delay is established in terms of LMIs, which allow simultaneous computation of two bounds that characterize the exponential stability rate of the solution and can be easily determined by utilizing MATLABs LMI Control Toolbox.

The outline of the paper is as follows. Section 2 presents definitions and some well-known technical propositions needed for the proof of the main result. LMI delay-dependent exponential stability criteria for switched neural networks with interval time-varying delay criteria is presented in Section 3. The paper ends with conclusions and cited references.

\section{Preliminaries}

The following notation will be used in this paper. $\mathbb{R}^{+}$denotes the set of all real non-negative numbers; $\mathbb{R}^{n}$ denotes the $n$-dimensional space with the scalar product $\langle x, y\rangle$ or $x^{T} y$ of two vectors $x, y$, and the vector norm $\|.\| ; M^{n \times r}$ denotes the space of all matrices of $(n \times r)$-dimensions. $A^{T}$ denotes the transpose of matrix $A ; A$ is symmetric if $A=A^{T} ; I$ denotes the identity matrix; $\lambda(A)$ denotes the set of all eigenvalues of $A ; \lambda_{\max }(A)=\max \{\operatorname{Re} \lambda ; \lambda \in \lambda(A)\}$. $x_{t}:=\{x(t+s): s \in[-h, 0]\},\left\|x_{t}\right\|=\sup _{s \in[-h, 0]}\|x(t+s)\| ; C^{1}\left([0, t], \mathbb{R}^{n}\right)$ denotes the set of all $\mathbb{R}^{n}$-valued continuously differentiable functions on $[0, t]$; $L_{2}\left([0, t], \mathbb{R}^{m}\right)$ denotes the set of all the $\mathbb{R}^{m}$-valued square integrable functions on $[0, t]$;

Matrix $A$ is called semi-positive definite $(A \geq 0)$ if $\langle A x, x\rangle \geq 0$, for all $x \in \mathbb{R}^{n} ; A$ is positive definite $(A>0)$ if $\langle A x, x\rangle>0$ for all $x \neq 0 ; A>B$ means $A-B>0$. The notation $\operatorname{diag}\{\ldots\}$ stands for a block-diagonal matrix. The symmetric term in a matrix is denoted by $*$.

Consider the following switched neural networks with interval time-varying 
delay:

$$
\begin{aligned}
& \dot{x}(t)=A_{\gamma(x(t)} x(t)+W_{0 \gamma(x(t))} f(x(t))+W_{1 \gamma(x(t))} g(x(t-h(t))), \quad t \geq 0, \\
& x(t)=\phi(t), t \in\left[-h_{1}, 0\right]
\end{aligned}
$$

where $x(t)=\left[x_{1}(t), x_{2}(t), \ldots, x_{n}(t)\right]^{T} \in \mathbb{R}^{n}$ is the state of the neural, $u(.) \in$ $L_{2}\left([0, t], \mathbb{R}^{m}\right)$ is the control; $n$ is the number of neurals, and

$$
\begin{aligned}
& f(x(t))=\left[f_{1}\left(x_{1}(t)\right), f_{2}\left(x_{2}(t)\right), \ldots, f_{n}\left(x_{n}(t)\right)\right]^{T}, \\
& g(x(t))=\left[g_{1}\left(x_{1}(t)\right), g_{2}\left(x_{2}(t)\right), \ldots, g_{n}\left(x_{n}(t)\right)\right]^{T},
\end{aligned}
$$

are the activation functions; $\gamma():. R^{n} \rightarrow \mathcal{N}:=\{1,2, \ldots, N\}$ is the switching rule, which is a function depending on the state at each time and will be designed. A switching function is a rule which determines a switching sequence for a given switching system. Moreover, $\gamma(x(t))=j$ implies that the system realization is chosen as the $j^{\text {th }}$ system, $j=1,2, \ldots, N$. It is seen that the system (1) can be viewed as an autonomous switched system in which the effective subsystem changes when the state $x(t)$ hits predefined boundaries.

$A_{j}=\operatorname{diag}\left(\bar{a}_{1 j}, \bar{a}_{2 j}, \ldots, \bar{a}_{n j}\right), \bar{a}_{i j}>0$ represents the self-feedback term; $W_{0 j}$, $W_{1 j}$ denote the connection weights, the discretely delayed connection weights and the distributively delayed connection weight, respectively; The time-varying delay function $h(t)$ satisfies the condition

$$
0 \leq h_{0} \leq h(t) \leq h_{1},
$$

The initial functions $\phi(t) \in C^{1}\left(\left[-h_{1}, 0\right], R^{n}\right)$, with the norm

$$
\|\phi\|=\sup _{t \in\left[-h_{1}, 0\right]} \sqrt{\|\phi(t)\|^{2}+\|\dot{\phi}(t)\|^{2}} \text {. }
$$

In this paper we consider various activation functions and assume that the activation functions $f(),. g($.$) are Lipschitzian with the Lipschitz constants f_{i}, e_{i}>$ 0 :

$$
\begin{array}{ll}
\left|f_{i}\left(\xi_{1}\right)-f_{i}\left(\xi_{2}\right)\right| \leq f_{i}\left|\xi_{1}-\xi_{2}\right|, & i=1,2, \ldots, n, \forall \xi_{1}, \xi_{2} \in \mathbb{R}, \\
\left|g_{i}\left(\xi_{1}\right)-g_{i}\left(\xi_{2}\right)\right| \leq e_{i}\left|\xi_{1}-\xi_{2}\right|, & i=1,2, \ldots, n, \forall \xi_{1}, \xi_{2} \in \mathbb{R},
\end{array}
$$

Definition 1. Given $\alpha>0$. The zero solution of switched neural networks with interval time-varying delay (1) is $\alpha$-exponentially stable if there exist a positive number $N>0$ such that every solution $x(t, \phi)$ satisfies the following condition:

$$
\|x(t, \phi)\| \leq N e^{-\alpha t}\|\phi\|, \quad \forall t \geq 0
$$


We introduce the following technical well-known propositions, which will be used in the proof of our results.

Definition 2. The system of matrices $\left\{J_{i}\right\}, i=1,2, \ldots, N$, is said to be strictly complete if for every $x \in R^{n} \backslash\{0\}$ there is $i \in\{1,2, \ldots, N\}$ such that $x^{T} J_{i} x<0$.

It is easy to see that the system $\left\{J_{i}\right\}$ is strictly complete if and only if

$$
\bigcup_{i=1}^{N} \alpha_{i}=R^{n} \backslash\{0\},
$$

where

$$
\alpha_{i}=\left\{x \in R^{n}: \quad x^{T} J_{i} x<0\right\}, i=1,2, \ldots, N .
$$

We end this section with the following technical well-known propositions, which will be used in the proof of the main results.

Proposition 1. (Schur Complement Lemma, see [16]) Given constant matrices $X, Y, Z$ with appropriate dimensions satisfying $X=X^{T}, Y=Y^{T}>0$. Then $X+Z^{T} Y^{-1} Z<0$ if and only if

$$
\left(\begin{array}{cc}
X & Z^{T} \\
Z & -Y
\end{array}\right)<0
$$

Proposition 2. (Integral Matrix Inequality, see [16]) For any symmetric positive definite matrix $M>0$, scalar $\sigma>0$ and vector function $\omega:[0, \sigma] \rightarrow \mathbb{R}^{n}$ such that the integrations concerned are well defined, the following inequality holds

$$
\left(\int_{0}^{\sigma} \omega(s) d s\right)^{T} M\left(\int_{0}^{\sigma} \omega(s) d s\right) \leq \sigma\left(\int_{0}^{\sigma} \omega^{T}(s) M \omega(s) d s\right) .
$$

Proposition 3. (see [16]) The system $\left\{J_{i}\right\}, i=1,2, \ldots, N$, is strictly complete if there exist $\delta_{i} \geq 0, i=1,2, \ldots, N, \sum_{i=1}^{N} \delta_{i}>0$ such that

$$
\sum_{i=1}^{N} \delta_{i} J_{i}<0 .
$$

If $N=2$ then the above condition is also necessary for the strict completeness. 


\section{Main Results}

Let us set

$$
\begin{aligned}
& J_{i}=-P-\alpha A_{j}-\alpha A_{j}^{T}-A_{j}^{T} P-P A_{j}, \\
& \alpha_{i}=\left\{x \in R^{n}: \quad x^{T} J_{i} x<0\right\}, \quad i=1,2, \ldots, N, \\
& \bar{\alpha}_{1}=\alpha_{1}, \quad \bar{\alpha}_{i}=\alpha_{i} \backslash \bigcup_{j=1}^{i-1} \bar{\alpha}_{j}, \quad i=2,3, \ldots, N, \\
& w_{11}=-P-\alpha A_{j}, w_{12}=P+A_{j} P, w_{13}=A_{j} P, w_{14}=A_{j} P, \\
& w_{15}=P+A_{j} P, w_{22}=\left(h_{1}-h_{0}\right) U-2 P, w_{23}=P, w_{24}=P, w_{25}=P, \\
& w_{33}=-e^{-2 \alpha h_{1}} U, w_{34}=0, w_{35}=-2 \alpha h_{1} U, w_{44}=-e^{-2 \alpha h_{1}} U, w_{45}=e^{-2 \alpha h_{1}} U, \\
& w_{55}=-2 e^{-2 \alpha h_{1}} U, E=\operatorname{diag}\left\{e_{i}, i=1, \ldots, n\right\}, F=\operatorname{diag}\left\{f_{i}, i=1, \ldots, n\right\}, \\
& \lambda_{1}=\lambda_{\min }\left(P^{-1}\right), \lambda_{2}=\lambda_{\max }\left(P^{-1}\right)+h_{0} \lambda_{\max }\left[P^{-1}\left(\sum_{i=0}^{1} G_{i}\right) P^{-1}\right] \\
& \quad+h_{1}^{2} \lambda_{\max }\left[P^{-1}\left(\sum_{i=0}^{1} H_{i}\right) P^{-1}\right]+\left(h_{1}-h_{0}\right) \lambda_{\max }\left(P^{-1} U P^{-1}\right) .
\end{aligned}
$$

Theorem 1. Given $\alpha>0$. The zero solution of the switched neural networks with interval time-varying delay (1) is $\alpha$-exponentially stable if there exist symmetric positive definite matrices $P, U$, satisfying the following $L M I_{s}$

$$
\begin{gathered}
\text { (i) } \quad \mathcal{E}_{j}=\left[\begin{array}{ccccc}
w_{11} & w_{12} & w_{13} & w_{14} & w_{15} \\
* & w_{22} & w_{23} & w_{24} & w_{25} \\
* & * & w_{33} & w_{34} & w_{35} \\
* & * & * & w_{44} & w_{45} \\
* & * & * & * & w_{55}
\end{array}\right]<0, \quad j=1,2, \ldots, N, \\
\text { (ii) } \exists \delta_{i} \geq 0, i=1,2, \ldots, N, \quad \sum_{i=1}^{N} \delta_{i}>0: \sum_{i=1}^{N} \delta_{i} J_{i}<0,
\end{gathered}
$$

the switching rule is chosen as $\gamma(x(t))=j$. Moreover, the solution $x(t, \phi)$ of the system satisfies

$$
\|x(t, \phi)\| \leq \sqrt{\frac{\lambda_{2}}{\lambda_{1}}} e^{-\alpha t}\|\phi\|, \quad \forall t \geq 0 .
$$


Proof. Let $Y=P^{-1}, y(t)=Y x(t)$. We consider the following LyapunovKrasovskii functional

$$
V\left(t, x_{t}\right)=\sum_{i=1}^{2} V_{i}\left(t, x_{t}\right)
$$

$$
\begin{aligned}
& V_{1}=x^{T}(t) Y x(t), \\
& V_{2}=\left(h_{1}-h_{0}\right) \int_{t-h_{1}}^{t-h_{0}} \int_{t+s}^{t} e^{2 \alpha(\tau-t)} \dot{x}^{T}(\tau) Y U Y \dot{x}(\tau) d \tau d s .
\end{aligned}
$$

It easy to check that

$$
\lambda_{1}\|x(t)\|^{2} \leq V\left(t, x_{t}\right) \leq \lambda_{2}\left\|x_{t}\right\|^{2}, \quad \forall t \geq 0
$$

Taking the derivative of $V_{i}, \quad i=1,2$ we have

$$
\begin{aligned}
\dot{V}_{1} & =2 x^{T}(t) Y \dot{x}(t) \\
& =y^{T}(t)\left[-P A_{j}^{T}-A_{j} P\right] y(t)+2 y^{T}(t) W_{0 j} f(.) y(t)+2 y^{T}(t) W_{1 j} g(.) y(t) ; \\
\dot{V}_{2} & =\left(h_{1}-h_{0}\right)^{2} \dot{y}^{T}(t) U \dot{y}(t)-\left(h_{1}-h_{0}\right) e^{-2 \alpha h_{1}} \int_{t-h_{1}}^{t-h_{0}} \dot{y}^{T}(s) U \dot{y}(s) d s-2 \alpha V_{2} .
\end{aligned}
$$

Applying Proposition 2 and the Leibniz - Newton formula

$$
\int_{s}^{t} \dot{y}(\tau) d \tau=y(t)-y(s)
$$

we have for $j=1,2, i=0,1$ :

$$
\int_{t-h_{1}}^{t-h_{0}} \dot{y}^{T}(s) U \dot{y}(s) d s=\int_{t-h_{1}}^{t-h(t)} \dot{y}^{T}(s) U \dot{y}(s) d s+\int_{t-h(t)}^{t-h_{0}} \dot{y}^{T}(s) U \dot{y}(s) d s .
$$

Applying Proposition 2 gives

$$
\begin{aligned}
{\left[h_{1}-h(t)\right] \int_{t-h_{1}}^{t-h(t)} \dot{y}^{T}(s) U \dot{y}(s) d s } & \geq\left[\int_{t-h_{1}}^{t-h(t)} \dot{y}(s) d s\right]^{T} U\left[\int_{t-h_{1}}^{t-h(t)} \dot{y}(s) d s\right] \\
\geq & {\left[y ( t - h ( t ) - y ( t - h _ { 1 } ) ] ^ { T } U \left[y\left(t-h(t)-y\left(t-h_{1}\right)\right]\right.\right.}
\end{aligned}
$$

Since $h_{1}-h(t) \leq h_{1}-h_{0}$, we have

$$
\left[h_{1}-h_{0}\right] \int_{t-h_{1}}^{t-h(t)} \dot{y}^{T}(s) U \dot{y}(s) d s \geq\left[y ( t - h ( t ) - y ( t - h _ { 1 } ) ] ^ { T } U \left[y\left(t-h(t)-y\left(t-h_{1}\right)\right]\right.\right.
$$


then

$-\left[h_{1}-h_{0}\right] \int_{t-h_{1}}^{t-h(t)} \dot{y}^{T}(s) U \dot{y}(s) d s \leq-\left[y\left(t-h(t)-y\left(t-h_{1}\right)\right]^{T} U\left[y\left(t-h(t)-y\left(t-h_{1}\right)\right]\right.\right.$.

Similarly, we have

$-\left(h_{1}-h_{0}\right) \int_{t-h(t)}^{t-h_{0}} \dot{y}^{T}(s) U \dot{y}(s) d s \leq-\left[y\left(t-h_{0}\right)-y(t-h(t))\right]^{T} U\left[y\left(t-h_{0}\right)-y(t-h(t)]\right.$.

Then, we have

$$
\begin{aligned}
\dot{V}(.)+2 \alpha V(.) & \leq y^{T}(t)\left[-P A_{j}^{T}-A_{j} P\right] y(t)+2 y^{T}(t) W_{0 j} f(.)+2 y^{T}(t) W_{1 j} g(.) \\
& +2 \alpha\langle P y(t), y(t)\rangle+\left(h_{1}-h_{0}\right) \dot{y}^{T}(t) U \dot{y}(t) \\
& -e^{-2 \alpha h_{1}}\left[y(t-h(t))-y\left(t-h_{1}\right)\right]^{T} U\left[y(t-h(t))-y\left(t-h_{1}\right)\right] \\
& -e^{-2 \alpha h_{1}}\left[y\left(t-h_{0}\right)-y(t-h(t))\right]^{T} U\left[y\left(t-h_{0}\right)-y(t-h(t))\right] \\
& =x^{T}(t) J_{i} x(t)+\zeta^{T}(t) \mathcal{E}_{j} \zeta(t)
\end{aligned}
$$

where $\zeta(t)=\left[y(t), \dot{y}(t), y\left(t-h_{0}\right), y\left(t-h_{1}\right), y(t-h(t))\right]$. Therefore, we finally obtain from (5) and the condition (i) that

$$
\dot{V}(.)+2 \alpha V(.) \leq x^{T}(t) J_{i} x(t), \quad \forall i=1,2, \ldots, N, \quad t \in R^{+} .
$$

We now apply the condition (ii) and Proposition 3, the system $J_{i}$ is strictly complete, and the sets $\alpha_{i}$ and $\bar{\alpha}_{i}$ by (3) are well defined such that

$$
\begin{gathered}
\bigcup_{i=1}^{N} \alpha_{i}=R^{n} \backslash\{0\}, \\
\bigcup_{i=1}^{N} \bar{\alpha}_{i}=R^{n} \backslash\{0\}, \quad \bar{\alpha}_{i} \cap \bar{\alpha}_{j}=\emptyset, i \neq j .
\end{gathered}
$$

Therefore, for any $x(t) \in R^{n}, \quad t \in R^{+}$, there exists $i \in\{1,2, \ldots, N\}$ such that $x(t) \in \bar{\alpha}_{i}$. By choosing switching rule as $\gamma(x(t))=i$ whenever $\gamma(x(t)) \in \bar{\alpha}_{i}$, from (5) we have

$$
E[\dot{V}(.)+2 \alpha V(.)] \leq E\left[x^{T}(t) J_{i} x(t)\right]<0, \quad t \in R^{+},
$$

and hence

$$
\dot{V}\left(t, x_{t}\right) \leq-2 \alpha V\left(t, x_{t}\right), \quad \forall t \in R^{+}
$$


Integrating both sides of (6) from 0 to $t$, we obtain

$$
V\left(t, x_{t}\right) \leq V(\phi) e^{-2 \alpha t}, \quad \forall t \in R^{+} .
$$

Furthermore, taking condition (4) into account, we have

$$
\lambda_{1}\|x(t, \phi)\|^{2} \leq V\left(x_{t}\right) \leq V(\phi) e^{-2 \alpha t} \leq \lambda_{2} e^{-2 \alpha t}\|\phi\|^{2},
$$

then

$$
\|x(t, \phi)\| \leq \sqrt{\frac{\lambda_{2}}{\lambda_{1}}} e^{-\alpha t}\|\phi\|, \quad t \in R^{+},
$$

which concludes the proof by Definition 1, concludes the proof of the theorem.

\section{Conclusion}

In this paper, the problem of exponential stability for switched neural networks with interval nondifferentiable time-varying delay has been studied. By constructing a set of time-varying Lyapunov-Krasovskii functional combined with Newton-Leibniz formula, a switching rule and switching design for exponential stability of switched neural networks with interval time-varying delay have been presented and new sufficient conditions for the exponential stability for the system have been derived in terms of LMIs.

\section{Acknowledgments}

This work was supported by the Thailand Research Fund Grant, the Commission for Higher Education and Faculty of Science, Maejo University, Thailand. The authors thank anonymous reviewers for valuable comments and suggestions, which allowed us to improve the paper.

\section{References}

[1] Hopfield J.J., "Neural networks and physical systems with emergent collective computational abilities," Proc. Natl. Acad. Sci. USA, 79(1982), 2554-2558.

[2] K. Ratchagit, Asymptotic stability of delay-difference system of Hopfield neural networks via matrix inequalities and application, International Journal of Neural Systems, 17(2007), 425-430. DOI: 10.1142/S0129065707001263 
[3] J.H. Park, Further result on asymptotic stability criterion of cellular neural networks with time-varying discrete and distributed delays, Applied Mathematics and Computation, 182(2006), 1661-1666.

[4] K. Ratchagit and V. N. Phat, Robust Stability and Stabilization of Linear Polytopic Delay-Difference Equations with Interval Time-Varying Delays, Neural, Parallel, and Scientific Computations, 19(2011), 361-372.

[5] V. N. Phat and K. Ratchagit, Stability and stabilization of switched linear discrete-time systems with interval time-varying delay, Nonlinear Analysis: Hybrid Systems, 5(2011), 605-612. DOI: 10.1016/j.nahs.2011.05.006

[6] VN. Phat, Y. Kongtham, and K. Ratchagit, LMI approach to exponential stability of linear systems with interval time-varying delays, Linear Algebra Appl., Vol. 436, pp. 243-251, 2012. doi: 10.1016/j.laa.2011.07.016

[7] Kreangkri Ratchagit, STABILITY ANALYSIS OF LINEAR SYSTEMS WITH TIME DELAYS, International Journal of Pure and Applied Mathematics, Vol. 76 No. 1, 2012, 21-28.

[8] M. de la Sen and A. Ibeas, Stability Results for Switched Linear Systems with Constant Discrete Delays, Mathematical Problems in Engineering, vol. 2008, Article ID 543145, 28 pages, 2008. doi:10.1155/2008/543145

[9] K. Ratchagit , THE SUFFICIENT CONDITIONS FOR STABILITY OF LINEAR TIME-VARYING SYSTEMS WITH STATE DELAYS, International Journal of Pure and Applied Mathematics, Vol. 65 No. 1, 2010, $65-72$.

[10] K. Ratchagit and V.N. Phat, Stability criterion for discrete-time systems, J. Ineq. Appl., 2010(2010), 1-6. doi:10.1155/2010/201459

[11] Grienggrai Rajchakit, Delay-Dependent Asymptotical Stabilization Criterion of Recurrent Neural Networks, Applied Mechanics and Materials. 330(2013) 1045-1048. doi:10.4028/www.scientific.net/AMM.330.1045

[12] K. Ratchagit, Asymptotic stability of nonlinear delay-difference system via matrix inequalities and application, International Journal of Computational Methods, pp. 389-397, 2009. DOI: 10.1142/S0219876209001899

[13] Manlika Rajchakit and Grienggrai Rajchakit, Mean Square Exponential Stability of Stochastic Switched System with Interval Time-Varying Delays, Abstract and Applied Analysis, vol. 2012, Article ID 623014, 12 pages, 2012. doi:10.1155/2012/623014 
[14] M Rajchakit, G Rajchakit, Mean square robust stability of stochastic switched discrete-time systems with convex polytopic uncertainties, Journal of Inequalities and Applications 2012, 2012:135. doi:10.1186/1029242X-2012-135

[15] M. Rajchakit and G. Rajchakit, LMI approach to robust stability and stabilization of nonlinear uncertain discrete-time systems with convex polytopic uncertainties, Advances in Difference Equations, 2012(2012). doi:10.1186/1687-1847-2012-106

[16] R.P. Agarwal, Difference Equations and Inequalities, Second Edition, Marcel Dekker, New York, 2000. 
